\title{
Control and Disturbance Rejection for Discrete Linear Repetitive Processes
}

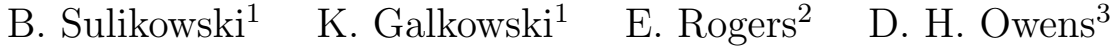 \\ ${ }^{1}$ Institute of Control and Computation Engineering, \\ University of Zielona Gora, Poland. \\ ${ }^{2}$ School of Electronics and Computer Science, \\ University of Southampton, Southampton SO17 1BJ, UK. \\ ${ }^{3}$ Department of Automatic Control and Systems Engineering, \\ University of Sheffield, Sheffield S1 3JD, UK.
}

\begin{abstract}
Repetitive processes are a distinct class of $2 \mathrm{D}$ systems (i.e. information propagation in two independent directions) of both systems theoretic and applications interest. They cannot be controlled by direct extension of existing techniques from either standard (termed 1D here) or 2D systems theory. Here we give new results on the relatively open problem of the design of physically based feedforward/feedback control laws to achieve desired performance and disturbance decoupling in the sense defined in the body of the paper.
\end{abstract}

Keywords: Repetitive processes, control, disturbance rejection, feedforward/feedback control.

\section{Introduction}

Linear repetitive processes are a distinct class of 2D systems of both system theoretic and applications interest. The essential unique characteristic of such a process is a series of sweeps, termed passes, through a set of dynamics defined over a fixed finite duration known as the pass length. On each pass an output, termed the pass profile, is produced which acts as a forcing function on, and hence contributes to, the next pass profile. This, in turn, leads to the unique control problem for these processes in that the output sequence of pass profiles generated can contain oscillations that increase in amplitude in the pass-to-pass direction.

To introduce a formal definition, let $\alpha<+\infty$ denote the pass length (assumed constant). Then in a repetitive process the pass profile $y_{k}(p), 0 \leq p \leq \alpha$, generated on pass $k$ acts as a forcing function on, and hence contributes to, the next pass profile $y_{k+1}(p), 0 \leq p<\alpha, k \geq 0$. The fact that the pass length is finite (and hence information in this direction only occurs over a finite duration) is the key difference with other classes of $2 \mathrm{D}$ linear systems, such as those with discrete dynamics described by the well known and extensively studied Roesser [12] and Fornasini Marchesini [6] state space models.

Physical examples of repetitive processes include long-wall coal cutting and metal rolling operations (see, for example, $[5,3]$ ). Also in recent years applications have arisen where adopting a repetitive process setting for analysis has distinct advantages over alternatives. Examples of these so-called algorithmic applications of repetitive processes include classes of iterative learning control (ILC) schemes [1] and iterative algorithms for solving nonlinear dynamic optimal control problems based on the maximum principle [11]. In the case of ILC for the linear dynamics case, the stability theory for differential and discrete linear repetitive processes is the essential basis for a rigorous stability/convergence analysis of such algorithms.

A rigorous stability theory for linear repetitive processes has been developed. This theory [13] is based on an abstract model in a Banach space setting which includes all such processes as special cases. In essence, this stability theory is a form of bounded-input bounded-output stability which prevents 
the appearance of oscillations in the output pass profile sequence in the pass-to-pass direction and also, in its strongest form, ensures that the dynamics along a pass are also bounded independent of the pass length. Also the results of applying this theory to a wide range of sub-classes of such processes have been reported, including the one required here where the resulting conditions can be tested by direct application of well known 1D linear systems tests.

It is possible define physically meaningful control laws for repetitive processes where in the ILC application, for example, one such family of control laws is composed of (state or output based) feedback control action on the current pass combined with information 'feedforward' from the previous pass (or trial in the ILC context) which, of course, has already been generated and is therefore available for use. The majority of the work to date on the control of linear repetitive processes (outside the ILC application area where extra structure arises which can be used to advantage) has been on ensuring stability under control action - see, for example, [9]. In this paper, we develop new results on the design of control laws for performance and disturbance rejection (in addition to stability).

Next we introduce the sub-class of constant pass length linear repetitive processes considered here and other necessary background. Throughout this paper, the identity matrix with the required dimensions is denoted by $I$, respectively. Moreover, $M>0$ denotes a real symmetric positive definite matrix.

\section{Background}

The state space model of the discrete linear repetitive processes considered in this paper has the following form over $0 \leq p \leq \alpha-1, k \geq 0$

$$
\begin{aligned}
x_{k+1}(p+1) & =A x_{k+1}(p)+B u_{k+1}(p)+B_{0} y_{k}(p)+E w_{k+1}(p) \\
y_{k+1}(p) & =C x_{k+1}(p)+D u_{k+1}(p)+D_{0} y_{k}(p)+F w_{k+1}(p)
\end{aligned}
$$

Here on pass $k, x_{k}(p)$ is the $n \times 1$ state vector, $y_{k}(p)$ is the $m \times 1$ pass profile vector, $u_{k}(p)$ is the $q \times 1$ vector of control inputs, and $w_{k+1}(p)$ is a $f \times 1$ disturbance vector which enters on the current pass and here is assumed to be the same value on each pass and denoted from this point onwards by $w(p)$.

This state space model is a natural extension of that first introduced in [13] to allow for disturbances which enter both the state and pass profile dynamics on each pass. To complete the process description, it is necessary to specify the 'initial conditions' - termed the boundary conditions here, i.e. the state initial vector on each pass and the initial pass profile. Here these are taken to be The simplest possible choice for these is

$$
\begin{aligned}
x_{k+1}(0) & =d_{k+1}, k \geq 0 \\
y_{0}(t) & =f(p)
\end{aligned}
$$

where the $n \times 1$ vector $d_{k+1}$ has known constant entries and $f(p)$ is an $m \times 1$ vector whose entries are known functions of $p$ over $0 \leq p \leq \alpha-1$.

The first attempt to control these processes [5] arose in the long-wall coal cutting operation and used the standard Nyquist criteria for continuous time systems (with independent variable $t$ ). To apply this, an auxiliary independent variable termed the total distance traversed was first used to convert the process dynamics into to those of an equivalent infinite length single pass process. At point $t$ on pass $k+1$ this variable, denoted by $v$ here, is given by $v=k \alpha+t$ and hence, for example, $y_{k+1}(t)$ is transformed to $y(v)$. Once this task was completed, the Laplace transform was applied to the resulting process dynamics to obtain a transfer function and the assertion then was that the original repetitive process was stable if, and only if, its infinite length single pass equivalent had this property.

In effect, this stability analysis ignored the fact that the pass initial conditions are reset before the start of each new pass and also destroyed the essential finite pass length repeatable nature of the underlying dynamics. This, in turn, led to a new stability theory [13] for linear constant pass length repetitive processes which is based on an abstract model of the process dynamics in a Banach space (here denoted by $E_{\alpha}$ ) of the form

$$
y_{k+1}=L_{\alpha} y_{k}+b_{k+1}, k \geq 0
$$


In this model $y_{k} \in E_{\alpha}$ is the pass profile on pass $k, L_{\alpha}$ is a bounded linear operator mapping $E_{\alpha}$ into itself and $b_{k+1} \in W_{\alpha}$, where $W_{\alpha}$ is a linear subspace of $E_{\alpha}$. Also the term $L_{\alpha} y_{k}$ describes the contribution of pass $k$ to pass $k+1$ and $b_{k+1}$ represents control inputs and other effects which enter on the current pass.

The unique control problem for these processes is that the output sequence of pass profiles $\left\{y_{k}\right\}_{k \geq 1}$ can contain oscillations which increase in amplitude in the pass-to-pass direction. Hence a natural definition of stability is to demand bounded input sequences produce bounded sequences of outputs (in this case pass profiles). Suppose therefore that $\|\cdot\|$ denotes the norm on $E_{\alpha}$. Then so-called asymptotic stability holds provided there exist real numbers $M_{\alpha}>0$ and $\lambda_{\alpha} \in(0,1)$ such that $\left\|L_{\alpha}^{k}\right\| \leq M_{\alpha} \lambda_{\alpha}^{k}, k \geq 0$ (where $\|\cdot\|$ is also used to denote the induced operator norm). This property requires that the spectral radius, $r\left(L_{\alpha}\right)$, of $L_{\alpha}$ satisfies $r\left(L_{\alpha}\right)<1$.

To establish conditions for asymptotic stability of a particular example therefore requires the computation of the spectral values of the corresponding $L_{\alpha}$. Also if asymptotic stability holds then $\left\{y_{k}\right\}_{k \geq 1}$ converges strongly (in the pass-to-pass direction) to the so-called steady, or limit, profile $y_{\infty}$ which is the unique solution of the linear equation

$$
y_{\infty}=L_{\alpha} y_{\infty}+b_{\infty}
$$

In the case of processes described by (1), a simple extension of the result for the case [13] when $w(p)=0$ yields that asymptotic stability holds if, and only if, $r\left(D_{0}\right)<1$ and the resulting limit profile is described by the $1 \mathrm{D}$ linear system

$$
\begin{aligned}
x_{\infty}(p+1) & =\left(A+B_{0}\left(I-D_{0}\right)^{-1} C\right) x_{\infty}(p)+B u_{\infty}(p)+E\left(I-D_{0}\right)^{-1} F w(p), x_{\infty}(0)=d_{s} \\
y_{\infty}(p) & =\left(I-D_{0}\right)^{-1}\left[C x_{\infty}(p)+F w(p)\right]
\end{aligned}
$$

where $d_{s}$ denotes the strong limit of the pass state initial vector sequence $\left\{d_{k+1}\right\}_{k \geq 0}$. A key fact, however, is that this property does not guarantee that the limit profile has 'acceptable' along the pass dynamics where the most basic requirement is stability in the $1 \mathrm{D}$ sense, i.e. $r\left(A+B_{0}\left(I_{m}-D_{0}\right)^{-1} C\right)<1$ - a point which is easily illustrated by, for example, the case when $A=-0.5, B=0, B_{0}=0.5+b_{0}, C=$ $1, D=D_{0}=0$, and the real scalar $b_{0}$ is chosen such that $\left|b_{0}\right| \geq 1$.

The reason why asymptotic stability does not guarantee a limit profile which is 'stable along the pass' is due to the finite pass length. In particular, asymptotic stability is easily shown to be boundedinput bounded-output (BIBO) stability with respect to the finite and fixed pass length. Also in cases where a limit profile which is unstable as a $1 \mathrm{D}$ linear system is not acceptable, the stronger concept of stability along the pass must be used which, in effect, demands the BIBO property uniformly, i.e. independent of the pass length. In terms of the abstract model, stability along the pass holds provided there exists real numbers $M_{\infty}>0$ and $\lambda_{\infty} \in(0,1)$ which are independent of $\alpha$ such that $\left\|L_{\alpha}^{k}\right\| \leq M_{\infty} \lambda_{\infty}^{k}, k \geq 0$.

In the case of processes described by (1) several equivalent sets of necessary and sufficient conditions for stability along the pass can be derived but here it is the following one which is required. The proof of this result follows immediately from that in [13] for the case of processes described by (1) with $w(p)=0$ and hence it is omitted here.

Theorem 1 Discrete linear repetitive processes described by (1) and (2) are stable along the pass if, and only if, the $2 D$ characteristic polynomial

$$
\mathcal{C}\left(z_{1}, z_{2}\right):=\operatorname{det}\left[\begin{array}{cc}
I-z_{1} A & -z_{1} B_{0} \\
-z_{2} C & I-z_{2} D_{0}
\end{array}\right] \neq 0 \text { in } \bar{U}^{2}
$$

where $\bar{U}^{2}=\left\{\left(z_{1}, z_{2}\right):\left|z_{1}\right| \leq 1,\left|z_{2}\right| \leq 1\right\}$.

Note here that necessary conditions for stability along the pass are $r\left(D_{0}\right)<1$ and $r(A)<1$. Hence asymptotic stability is a necessary condition for stability along the pass. Moreover, simply requiring that the dynamics along any pass are bounded independent of the pass length, i.e. $r(A)<1$, is also 
only a necessary condition for stability along the pass, as the simple example given above demonstrates. Consider also a 2D Roesser model whose state dynamics is governed by the so-called augmented plant matrix

$$
\left[\begin{array}{l|l}
A & B_{0} \\
\hline C & D_{0}
\end{array}\right]
$$

Then stability along the pass is equivalent to BIBO stability of this Roesser model (see the relevant references cited in [13]). This last fact enables stability tests to be exchanged between these two areas.

As noted in the introduction, repetitive processes arise in both physical examples (recently another application has arisen in self-servo writing in disk drives [10]) and in the so-called algorithmic applications already referred to in the introduction to this paper. Hence it is essential to complement the work on stability and systems theoretic properties with the development of control schemes. Only when such schemes are available (together with supporting design and performance assessment algorithms) can the true potential of repetitive processes be determined.

It is in this last respect that the analysis of discrete linear repetitive processes and 2D linear systems described by the Roesser model diverge. In the case of repetitive processes, a clear design objective is to obtain a limit profile with acceptable along the pass properties independent of the presence of disturbances. Such a design objective has no 2D Roesser model equivalent.

In the reminder of this paper we give significant new results on controller design for the discrete linear repetitive processes considered here and illustrate them using the particular case when

$$
\begin{gathered}
A=\left[\begin{array}{cccc}
1.9118 & -0.0047 & -1.4706 & 0.7353 \\
1 & 0 & 0 & 0 \\
0 & 0 & 0 & 0 \\
0 & 0 & 1 & 0
\end{array}\right], B=\left[\begin{array}{c}
-2.2059 \times 10^{-5} \\
0 \\
0 \\
0
\end{array}\right], B_{0}=\left[\begin{array}{c}
0.7794 \\
0 \\
1 \\
0
\end{array}\right] \\
E=\left[\begin{array}{l}
1 \\
0 \\
0 \\
0
\end{array}\right], C=\left[\begin{array}{lll}
1.9118-0.0047 & -1.4706 & 0.7353
\end{array}\right] \\
D=2.2059 \times 10^{-5}, D_{0}=0.7794, F=1
\end{gathered}
$$

This data comes from a model of a physical process which has been used previously in the $2 \mathrm{D}$ systems literature [14] where here we take $\alpha=20$.

\section{Control for stability and performance in the absence of distur- bances}

Clearly the most basic property for discrete linear repetitive processes is asymptotic stability and indeed in some applications this is all that can be achieved. For example, the matrix corresponding to $A$ in the optimal control application never satisfies $r(A)<1$ [11]. In general, however, stability along the pass will be required. This section considers the use of control laws to achieve asymptotic and/or stability along the pass coupled with desired pass-to-pass and along the pass performance as required. In the case of the asymptotic stability based analysis, we make use of the $1 \mathrm{D}$ equivalent model of the underlying dynamics which has is summarized next. It is essential to note at this stage that the 1D equivalent model does not (as with the approach of [5]) destroy the underlying finite pass length repeatable nature of the underlying dynamics and should be regarded as a 'reversible transformation to aid analysis'. Note also that the stability results given in this and the next sub-section also hold in the presence of the disturbance vector $w(p)$.

The 1D equivalent state space model for discrete linear repetitive processes in the absence of disturbances [8] has already found major use in control related analysis. To extend the equivalent 1D model to processes described by (1), first introduce the substitutions

$$
l=k+1, v_{l}(p)=y_{l-1}(p), 0 \leq p \leq \alpha-1
$$


Next, introduce the so-called global state, input, pass profile and disturbance vectors of dimensions $n \alpha \times 1, q \alpha \times 1, m \alpha \times 1$ and $f \alpha \times 1$ respectively

$$
X(l)=\left[\begin{array}{c}
x_{l}(1) \\
x_{l}(2) \\
x_{l}(3) \\
\vdots \\
x_{l}(\alpha)
\end{array}\right], U(l)=\left[\begin{array}{c}
u_{l}(0) \\
u_{l}(1) \\
u_{l}(2) \\
\vdots \\
u_{l}(\alpha-1)
\end{array}\right], V(l)=\left[\begin{array}{c}
v_{l}(0) \\
v_{l}(1) \\
v_{l}(2) \\
\vdots \\
v_{l}(\alpha-1)
\end{array}\right], W=\left[\begin{array}{c}
w(0) \\
w(1) \\
w(2) \\
\vdots \\
w(\alpha-1)
\end{array}\right]
$$

Then the 1D equivalent state space model of the dynamics of (1) takes the form

$$
\begin{aligned}
X(l) & =\Gamma V(l)+\Sigma U(l)+\Psi_{0} d_{l}+\Omega_{x} W \\
V(l+1) & =\Phi V(l)+\Delta U(l)+\Theta_{0} d_{l}+\Omega_{y} W
\end{aligned}
$$

where for the purposes of this work it is only necessary to detail the structure of the following matrices

$$
\begin{gathered}
\Delta=\left[\begin{array}{ccccc}
D & 0 & 0 & \ldots & 0 \\
C B & D & 0 & \ldots & 0 \\
C A B & C B & D & \ldots & 0 \\
\vdots & \vdots & \vdots & \ddots & \vdots \\
C A^{\alpha-2} B & C A^{\alpha-3} B & C A^{\alpha-4} B & \ldots & D
\end{array}\right], \Phi=\left[\begin{array}{ccccc}
D_{0} & 0 & 0 & \ldots & 0 \\
C B_{0} & D_{0} & 0 & \ldots & 0 \\
C A B_{0} & C B_{0} & D_{0} & \ldots & \vdots \\
\vdots & \vdots & \vdots & \ddots & 0 \\
C A^{\alpha-2} B_{0} & C A^{\alpha-3} B_{0} & C A^{\alpha-4} B_{0} & \ldots & D_{0}
\end{array}\right] \\
\Omega_{x}=\left[\begin{array}{ccccc}
E & 0 & 0 & \ldots & 0 \\
A E & E & 0 & \ldots & 0 \\
A^{2} E & A E & E & \ldots & 0 \\
\vdots & \vdots & \vdots & \ddots & \vdots \\
A^{\alpha-1} E & A^{\alpha-2} E & A^{\alpha-3} E & \ldots & E
\end{array}\right], \Omega_{y}=\left[\begin{array}{ccccc}
0 & 0 & \ldots & 0 \\
C E & F & 0 & \ldots & 0 \\
C A E & C E & F & \ldots & 0 \\
\vdots & \vdots & \vdots & \ddots & \vdots \\
C A^{\alpha-2} E & C A^{\alpha-3} E & C A^{\alpha-4} E & \ldots & F
\end{array}\right]
\end{gathered}
$$

These last two matrices describe the influence of the disturbance terms and will be required in Section 4.

\subsection{Asymptotic stability with performance design}

We consider the use of the following feedback control law expressed in terms of the $1 \mathrm{D}$ equivalent model as

$$
U(l)=K V(l)
$$

This control law is activated only by the previous pass profile. Hence it is to be expected that it will be of limited effectiveness in controlling the along the pass dynamics.

Using well known LMI results (see, for example, $[2,7]$ ) we immediately obtain the following result for asymptotic stability of the resulting closed loop process. This control law only requires knowledge of the previous pass profile which is available by definition of the process dynamics and can be precomputed before the start of each new pass. Note also that it has no (explicit) dependence on the process state dynamics which clearly govern the behavior along each pass (and hence stability along the pass).

Theorem 2 Suppose that the $1 D$ equivalent model is used to design a control law of the form (6) for a discrete linear repetitive process defined by (1) and (2) with $w(p)=0$. Then the resulting closed loop process is asymptotically stable if, and only if, there exist matrices $P>0, G$ and $L$ such that the following LMI is feasible

$$
\left[\begin{array}{cc}
-P & \Phi G+\Delta L \\
G^{T} \Phi^{T}+L^{T} \Delta^{T} & G+G^{T}-P
\end{array}\right]<0
$$

Also if this last condition holds then the controller matrix $K$ is given by

$$
K=L G^{-1}
$$


Next we give a new result which address the currently open question of how to design a control law for discrete linear repetitive processes for both closed loop stability and performance. We assume that the control law of (6) is applied and hence that no reference or tracking vector is specified for each current pass (analogous to 1D linear systems regulator theory). The problem then is controller design for asymptotic stability with the additional requirement that the resulting limit profile vector is identically zero over the pass length.

Model matching control is a long standing technique in standard (or 1D) systems theory and there has also been some work on this problem for $2 \mathrm{D}$ discrete linear systems described by the Roesser and Fornasini state space models [14]. In the case of the discrete linear repetitive processes treated here, first note that the system matrix $\Phi$ describes the contribution of the previous pass profile to the current one. Also under the action of the control law (6) this matrix is 'mapped' as follows

$$
\Phi \rightarrow \Phi+\Delta K
$$

Suppose now that we want to assign the closed loop matrix here to $\widehat{\Phi}$ where this matrix is selected to give a state space model whose behavior the controlled process is required to follow. Then we have the following result.

Theorem 3 Suppose that the 1D equivalent model is used to design a control law of the form (6) for a discrete linear repetitive process defined by (1) and (2) with $w(p)=0$. Then the resulting closed loop process is asymptotically stable and reaches the required form $\widehat{\Phi}$ if there exist matrices $P>0, G$ and $L$ such that the following $L M I$ is feasible

$$
\left[\begin{array}{cc}
-P & (\Phi-\widehat{\Phi}) G+\Delta L \\
G^{T}(\Phi-\widehat{\Phi})^{T}+L^{T} \Delta^{T} & G+G^{T}-P
\end{array}\right]<0
$$

If this condition holds, the control law matrix $K$ is computed using the formula (8) in such a way that

$$
\Phi-\widehat{\Phi}+\Delta K=0
$$

Proof. First note again that if the LMI (10) holds then the control law matrix $K$ of $(6)$ is given by (8). Also it is a standard fact that it is possible to obtain from the LMI solver a $K$ such that (11) holds. In such a case, the closed loop system matrix is

$$
\widehat{\Phi}=\Phi+\Delta K
$$

which completes the proof.

Suppose now that the matrix $K$ can be designed such that (11) of Theorem 3 holds. Then, since the control law does not contain a tracking or reference vector, the resulting limit profile is zero, i.e. the process has been regulated to produce zero output over the pass length on the limit profile.

It is essential to note here that it is impossible to obtain an arbitrarily specified model matrix $\widehat{\Phi}$ starting from an arbitrarily specified $\Phi$. However, conditions under which (11) has a solution can be characterized easily on the basis of, for example, Cramer's rule for linear vector equations and the matrix Kronecker product. This is clearly an area for further research and the remainder of this section focuses on the design of control laws for stability along the pass and desired performance, as opposed to just stability along the pass in the work reported to-date in this subject area.

\subsection{Stability along the pass with performance design}

Here we consider a control law of the form $0 \leq p \leq \alpha-1, k \geq 0$

$$
u_{k+1}(p)=K_{1} x_{k+1}(p)+K_{2} y_{k}(p):=\widetilde{K}\left[\begin{array}{c}
x_{k+1}(p) \\
y_{k}(p)
\end{array}\right]
$$

where $K_{1}$ and $K_{2}$ are appropriately dimensioned matrices to be designed. In effect, this control law uses feedback of the current pass state vector (which is assumed to be available for use) and 
'feedforward' of the previous pass profile vector (compare with the control law of the previous subsection). (Note that in the repetitive process literature the term 'feedforward' is used to describe the case where (state or pass profile) information from the previous pass (or passes) is used as (part of) the input to a control law applied on the current pass, i.e. to information which is propagated in the pass-to-pass ( $k$ ) direction.)

This control law has clear physical meaning for practical applications of discrete linear repetitive processes and the following result uses the LMI setting (in effect, Theorem 1 closed loop is replaced by a sufficient condition which directly yields a computable formula for the controller matrices) to give a controller design algorithm which can be easily implemented (for background on the required computations see, for example, [4]) and where

$$
\widehat{A}_{1}=\left[\begin{array}{cc}
A & B_{0} \\
0 & 0
\end{array}\right], \quad \widehat{A}_{2}=\left[\begin{array}{cc}
0 & 0 \\
C & D_{0}
\end{array}\right]
$$

Theorem 4 [7] Suppose that a control law of the form (12) is applied to a discrete linear repetitive process described by (1) and (2) with $w(p)=0$. Then the resulting closed loop process is stable along the pass if there exist matrices $Y>0, Z>0$, and $N$ such that the following LMI holds

$$
\left[\begin{array}{ccc}
Z-Y & 0 & Y \widehat{A}_{1}^{T}+N^{T} \widehat{B}_{1}^{T} \\
0 & -Z & Y \widehat{A}_{2}^{T}+N^{T} \widehat{B}_{2}^{T} \\
\widehat{A}_{1} Y+\widehat{B}_{1} N & \widehat{A}_{2} Y+\widehat{B}_{2} N & -Y
\end{array}\right]<0
$$

where

$$
\widehat{B}_{1}=\left[\begin{array}{c}
B \\
0
\end{array}\right], \widehat{B}_{2}=\left[\begin{array}{c}
0 \\
D
\end{array}\right]
$$

If (13) holds, then a stabilizing $\widetilde{K}$ in the control law (12) is given by

$$
\widetilde{K}=N Y^{-1}
$$

Note that the state space quadruple $\left\{A, B_{0}, C, D_{0}\right\}$ describes the contribution of the previous pass profile to the current one and also stability along the pass - see Theorem 1. Also under the action of the control law (12) this quadruple is 'mapped' as follows

$$
\left[\begin{array}{l|l}
A & B_{0} \\
\hline C & D_{0}
\end{array}\right] \rightarrow\left[\begin{array}{l|l}
A+B K_{1} & B_{0}+B K_{2} \\
\hline C+D K_{1} & D_{0}+D K_{2}
\end{array}\right]
$$

and since there is no closed loop tracking (or target) vector, the process closed loop dynamics is completely described by the matrices on the right-hand side of this last expression. Suppose also that we want to assign these closed loop matrices here to $\left\{\mathcal{A}, \mathcal{B}_{0}, \mathcal{C}, \mathcal{D}_{0}\right\}$, where these matrices are selected to give a state space model whose behavior the controlled process is required to follow. Then we have the following result.

Theorem 5 Suppose that a control law of the form (12) is applied to a discrete linear repetitive process described by (1) and (2) with $w(p)=0$. Suppose also that the procedure of (13) and (14) produces controller matrices $K_{1}, K_{2}$ such that

$$
\left[\begin{array}{c|c}
A-\mathcal{A} & B_{0}-\mathcal{B}_{0} \\
\hline C-\mathcal{C} & D_{0}-\mathcal{D}_{0}
\end{array}\right]+\left[\begin{array}{c}
B \\
\hline D
\end{array}\right]\left[K_{1} K_{2}\right]=0
$$

Then the resulting closed loop process is stable along the pass and its dynamics are defined by the (previously specified) state space quadruple $\left\{\mathcal{A}, \mathcal{B}_{0}, \mathcal{C}, \mathcal{D}_{0}\right\}$ if there exist matrices $P>0$ and $Q>0$ such that

$$
\left[\begin{array}{ccc}
Z-Y & 0 & Y \widetilde{A}_{1}^{T}+N^{T} \hat{B}_{1}^{T} \\
0 & -Z & Y \widetilde{A}_{2}^{T}+N^{T} \hat{B}_{2}^{T} \\
\widetilde{A}_{1} Y+\hat{B}_{1} N & \widetilde{A}_{2} Y+\hat{B}_{2} N & -Y
\end{array}\right]<0
$$


where

$$
\widetilde{A}_{1}=\left[\begin{array}{cc}
A-\mathcal{A} & B_{0}-\mathcal{B}_{0} \\
0 & 0
\end{array}\right], \widetilde{A}_{2}=\left[\begin{array}{cc}
0 & 0 \\
C-\mathcal{C} & D_{0}-\mathcal{D}_{0}
\end{array}\right]
$$

and the other matrices are as before. The control law matrices $K_{1}$ and $K_{2}$ are again computed using (14).

Proof. First note again that if the LMI of (13) holds then the control law matrix $\widetilde{K}=\left[K_{1} K_{2}\right]$ is given by (14). Also it is a standard fact that it is possible to obtain from the LMI solver a $\widetilde{K}$ such that (15) holds. In which case, the closed loop system matrices are such that

$$
\left[\begin{array}{l|l}
\tilde{A} & \tilde{B}_{0} \\
\hline \tilde{C} & \tilde{D}_{0}
\end{array}\right]:=\left[\begin{array}{l|l}
A+B K_{1} & B_{0}+B K_{2} \\
\hline C+D K_{1} & D_{0}+D K_{2}
\end{array}\right]=\left[\begin{array}{c|c}
\mathcal{A} & \mathcal{B}_{0} \\
\hline \mathcal{C} & \mathcal{D}_{0}
\end{array}\right]
$$

which completes the proof.

The problem encountered in the analysis of the previous sub-section also arises here. In particular, it is impossible to obtain an arbitrarily specified model matrices $\left\{\mathcal{A}, \mathcal{B}_{0}, \mathcal{C}, \mathcal{D}_{0}\right\}$ starting from an arbitrary specified $\left\{A, B_{0}, C, D_{0}\right\}$. However, conditions under which (15) has a solution can be characterized easily on the basis, for example, of Cramer's rule for linear vector equations and the matrix Kronecker product.

In common with 1D linear systems, a natural approach to the control of repetitive processes is to specify a reference, or tracking vector, representing desired performance on each pass and then attempt to use a control law to achieve this goal. Here we consider the case when the term $K_{3} r_{k+1}(p), 0 \leq$ $p \leq \alpha-1$ is added to the control law where $r_{k+1}(p)$ is an $m \times 1$ column vector representing desired behavior on pass $k+1, k \geq 0$, and $K_{3}$ is an $q \times m$ controller matrix to be selected. This results in the closed loop process state space model

$$
\begin{aligned}
x_{k+1}(p+1) & =\mathcal{A} x_{k+1}(p)+B K_{3} r_{k+1}(p)+\mathcal{B}_{0} y_{k}(p) \\
y_{k+1}(p) & =\mathcal{C} x_{k+1}(p)+D K_{3} r_{k+1}(p)+\mathcal{D}_{0} y_{k}(p)
\end{aligned}
$$

The questions which now arise are: (i) what is a suitable choice for $r_{k+1}(p)$ ?; and (ii) how can we design the control law to give stability along the pass plus acceptable transient behavior?

To illustrate what can be achieved here, we focus on the single-input single-output case and use the numerical data given earlier in this paper and select the current pass reference vector as $r_{k+1}(p)=-1,0 \leq p \leq \alpha-1, k \geq 0$, i. e. a downward unit step applied at $p=0$ on each pass.

One possible way of designing the control law is to note that $K_{3}$ does not influence stability along the pass. Hence we can execute the LMI design of Theorem 5 to obtain control law matrices $K_{1}$ and $K_{2}$ which ensure closed loop stability along the pass and then attempt to select a suitable $K_{3}$ to meet the performance requirements by 'tuning' the response of the resulting closed loop process model. In the case of the numerical example given in the previous section, it is easily checked that this model is unstable along the pass and the stabilization procedure of Theorem 5 gives the control law matrices $K_{1}$ and $K_{2}$ as

$$
K_{1}=\left[\begin{array}{llll}
83203.2 & 4226.3 & -67555.2 & 29928.8
\end{array}\right], K_{2}=[-2882.67]
$$

and in the resulting stable along the pass closed loop process (It was also found that $K_{3}=-3765.8$ gave acceptable performance.)

$$
\left[\begin{array}{l|l}
\mathcal{A} & \mathcal{B}_{0} \\
\hline \mathcal{C} & \mathcal{D}_{0}
\end{array}\right]=\left[\begin{array}{cccc|c}
0.0764 & -0.0979 & 0.0196 & 0.0751 & 0.8430 \\
1.0 & 0.0 & 0.0 & 0.0 & 0.0 \\
0.0 & 0.0 & 0.0 & 0.0 & 1.0 \\
0.0 & 0.0 & 1.0 & 0.0 & 0.0 \\
\hline 0.0764 & -0.0979 & 0.0196 & 0.0751 & 0.8430
\end{array}\right]
$$

The empirical nature of the above approach means that it is clearly not feasible in the general case. Instead, the method developed next can be used. 
This new approach is based on a (simple structure) re-formulation of the problem and is the first step which allows us to extend the design analysis to the case when external disturbances are present - see the next section. The starting point is the fact that control task here is to drive the process pass profiles to some prescribed reference vector $y_{r e f}(p), 0 \leq p \leq \alpha-1$, where it is an immediate consequence (see Section 2 in this paper) of the stability theory that if asymptotic stability holds then the pass profile sequence converges to a steady, or so-called limit, profile described for discrete linear repetitive processes by a 1D linear systems state space model. Here what we are aiming to do is to force the process to produce the limit profile $y_{r e f}(p)$.

To solve this last problem, introduce the modified output vector variable termed the incremental pass profile vector as

$$
\chi_{k}(p):=y_{k}(p)-y_{r e f}(p)
$$

Then it is clear that the design requirement here is equivalent to

$$
\chi_{k}(p) \rightarrow 0,0 \leq p \leq \alpha-1, k \rightarrow \infty
$$

Now replace the process state space model $(1)$ with $w(p)=0$ by the following one obtained from it by substitution using (19)

$$
\begin{aligned}
x_{k+1}(p+1) & =A x_{k+1}(p)+B_{0} \chi_{k}(p)+B u_{k+1}(p) \\
\chi_{k+1}(p) & =C x_{k+1}(p)+D_{0} \chi_{k}(p)+D u_{k+1}(p)
\end{aligned}
$$

and apply to it the following control law (which is clearly of the form (12), i.e. current pass state feedback augmented in this case by feedforward of the difference between $y_{k}(p)$ and $\left.y_{r e f}(p)\right)$

$$
\begin{aligned}
u_{k+1}(p) & =K_{1} x_{k+1}(p)+K_{2} \chi_{k}(p) \\
& =K_{1} x_{k+1}(p)+K_{2} y_{k}(p)-K_{2} y_{r e f}(p)
\end{aligned}
$$

Also choose the closed loop control law (21) to transform the process of (20) into the form of those for which Theorem 5 holds, i.e. $\left\{\mathcal{A}, \mathcal{B}_{0}, \mathcal{C}, \mathcal{D}_{0}\right\}$. Then it follows immediately from the above analysis that the resulting closed loop model must be stable along the pass and also that the resulting limit profile is the zero vector over $0 \leq p \leq \alpha-1$. Moreover,

$$
x_{k}(p) \rightarrow 0,0 \leq p \leq \alpha-1, k \rightarrow \infty
$$

which is a natural, and most frequently obtained, result when using the LMI based approach to controller design.

Given this control law and converting back to the original pass profile vector $y_{k}(p)$ we obtain the resulting closed loop state space model

$$
\begin{aligned}
x_{k+1}(p+1) & =\mathcal{A} x_{k+1}(p)+\mathcal{B}_{0} y_{k}(p)-\mathcal{B}_{0} y_{r e f}(p) \\
y_{k+1}(p) & =\mathcal{C} x_{k+1}(p)+\mathcal{D}_{0} y_{k}(p)+\left(I-\mathcal{D}_{0}\right) y_{r e f}(p)
\end{aligned}
$$

which is stable along the pass and whose limit pass profile, due to (16) and (22), is clearly equal to $y_{r e f}(p)$, i.e. the control task has been exactly achieved.

\section{Design for Disturbance Rejection}

\subsection{Asymptotic Stability}

The aim here is full decoupling of the influence of the disturbance $w(p)$ together with simultaneously driving the process to the required reference vector denoted in this sub-section by $V_{r e f}$. In this case, it means that the resulting limit profile defined in terms of the $1 \mathrm{D}$ equivalent model as $V_{\infty}:=V(l)$ 
must be the a-priori specified vector $V_{\text {ref }}$ or, in component form,

$$
V_{\text {ref }}:=\left[\begin{array}{c}
y_{r e f}(0) \\
y_{r e f}(1) \\
y_{r e f}(2) \\
\vdots \\
y_{r e f}(\alpha-1)
\end{array}\right]
$$

and note again that asymptotic stability is equivalent to $r(\Phi)<1$.

The overall design task at this stage is to achieve the following:

(i) asymptotic stability, and hence the limit profile exists,

(ii) the resulting limit profile equals the a-priori specified vector, i.e.

$$
V(l) \rightarrow V_{\text {ref }}, l \rightarrow \infty
$$

and;

(iii) complete decoupling of the effects of the disturbance vector $w(p)$ closed loop.

Suppose now that asymptotic stability holds, i.e. $r(\Phi)<1$, then

$$
V(l+1)-V(l) \rightarrow 0, l \rightarrow \infty
$$

which is equivalent to

$$
V(l+1)=V(l) \equiv V_{\text {ref }}, l \rightarrow \infty
$$

and hence the resulting limit profile is described in this representation by

$$
V_{\text {ref }}=\Phi V_{\text {ref }}+\Delta U_{\infty}+\Theta_{0} d_{\infty}+\Omega_{y} W
$$

where $U_{\infty}$ denotes the $1 \mathrm{D}$ equivalent model representation of the strong limit of the control sequence $\left\{u_{k}\right\}_{k \geq 1}$ applied to the process, and $d_{\infty}$ denotes the strong limit of the pass state initial vector sequence $\left\{d_{k}\right\}_{k \geq 1}$.

Subtracting (23) from the second equation in (5) yields

$$
V(l+1)-V_{\text {ref }}=\Phi\left(V(l)-V_{\text {ref }}\right)+\Delta\left(U(l)-U_{\infty}\right)+\Theta_{0}\left(d_{l}-d_{\infty}\right)
$$

Now introduce the so-called residual variables

$$
\begin{aligned}
\hat{V}(l+1) & =V(l+1)-V_{\text {ref }} \\
\hat{U}(l+1) & =U(l+1)-U_{\infty} \\
\hat{d}_{l} & =d_{l}-d_{\infty}
\end{aligned}
$$

Then (24) can be written as

$$
\hat{V}(l+1)=\Phi \hat{V}(l)+\Delta \hat{U}(l)+\Theta_{0} \hat{d}_{l}
$$

and the external disturbance vector $w(p)$ has been decoupled from the process dynamics.

If the process is open loop asymptotically unstable, then control action can be employed which, in effect, is a modified form of (6). In particular, apply the following control law to (25)

$$
\hat{U}(l)=K \hat{V}(l) \Rightarrow U(l)-U_{\infty}=K\left(V(l)-V_{r e f}\right)
$$

and choose $K$ such that asymptotic stability holds closed loop, i.e. $r(\Phi+\Delta K)<1$. Then it follows immediately that this control law gives the required limit profile and also complete decoupling of the disturbance vector $w(p)$. 
The only difficulty here is that to apply $U(l)$, i.e.

$$
U(l)=K V(l)-K V_{r e f}+U_{\infty}
$$

it is necessary to compute $U_{\infty}$ explicitly. To do this, rewrite (23) in the form

$$
\Delta U_{\infty}=(I-\Phi) V_{r e f}-\Omega_{y} W-\Theta_{0} d_{\infty}
$$

to obtain the condition which must hold for the control task to be achieved. This, in turn, means that not all choices for $V_{\text {ref }}$ can be achieved and, in particular, only those which satisfy

$$
\operatorname{rank}\left[\Delta \mid(I-\Phi) V_{\text {ref }}-\Omega_{y} W-\Theta_{0} d_{\infty}\right]=\operatorname{rank}[\Delta]
$$

are feasible.

It now follows immediately from the structure of $\Delta$ that if $D$ is a full row rank matrix then (27) is solvable and hence

$$
U_{\infty}=\Delta^{\sharp}\left((I-\Phi) V_{\text {ref }}-\Omega_{y} W-\Theta_{0} d_{\infty}\right)
$$

where $(\cdot)^{\sharp}$ denotes the pseudo inverse of $(\cdot)$. Note that, if $m=q$ i.e. $\Delta$ is square and nonsingular (since $D$ is assumed to be full rank) matrix, the pseudo inverse can be replaced by the matrix inverse. In which case $(29)$ becomes

$$
U_{\infty}=\Delta^{-1}\left((I-\Phi) V_{r e f}-\Omega_{y} W-\Theta_{0} d_{\infty}\right)
$$

Note that if the input, pass profile and disturbance are scalars then $D \neq 0$ means that (30) is valid in this case. In the general case, however, it will often be the case that the dimension of the pass profile vector exceeds that of the input vector and hence neither $D$ or $\Delta$ are full row rank matrices. As noted before, not all possible limit profile vectors can be achieved under this design. There is, however, a means of reducing the limitations this may impose. This is due to the fact that it may be possible to choose $d_{\infty}$ to ensure that (28) holds.

Return now to the numerical example used in this paper and suppose that to meet other performance requirements we must have

$$
\left[\begin{array}{c|c}
\mathcal{A} & \mathcal{B}_{0} \\
\hline \mathcal{C} & \mathcal{D}_{0}
\end{array}\right]=\left[\begin{array}{cccc|c}
0.0764 & -0.0979 & 0.0196 & 0.0751 & 0.8430 \\
1.0 & 0.0 & 0.0 & 0.0 & 0.0 \\
0.0 & 0.0 & 0.0 & 0.0 & 1.0 \\
0.0 & 0.0 & 1.0 & 0.0 & 0.0 \\
\hline 0.0764 & -0.0979 & 0.0196 & 0.0751 & 0.8430
\end{array}\right]
$$

and $y_{\text {ref }}(p)=-1,0 \leq p \leq \alpha-1$. Also we consider the case when $w(p)$ is the sampled sine wave $w(p)=-0.5 \sin \left(\frac{2 \pi(p-1)}{\alpha}\right)$.

The controller matrix $K$ in $(26)$ in this case is

$$
K=\left[\begin{array}{lllll}
K^{1} & K^{2} & K^{3} & \ldots & K^{20}
\end{array}\right]=\left[\begin{array}{ccccc}
k_{1} & 0 & 0 & \ldots & 0 \\
k_{2} & k_{1} & 0 & \ldots & 0 \\
k_{3} & k_{2} & k_{1} & \ldots & 0 \\
\vdots & \vdots & \vdots & \ddots & \vdots \\
k_{20} & k_{19} & k_{18} & \ldots & k_{1}
\end{array}\right]
$$

where

$$
\begin{aligned}
& K^{1}= {\left[\begin{array}{lllllllll}
-2882.6667 & 2585.0977 & 40481.0829 & 270.8555 & -694.9791 & \ldots \\
& \ldots & -79.6131 & 61.9560 & 12.5276 & -5.1084 & -1.6167 & \ldots \\
& \ldots & 0.3766 & 0.1870 & -0.0226 & -0.0200 & 0.0007 & \ldots & \\
& \ldots & 0.0020 & 0.0001 & -0.0002 & 0.0 & 0.0
\end{array}\right]^{T} } \\
&
\end{aligned}
$$




\subsection{Stability Along the Pass}

Now we show how to solve the design problem considered here under stability along the pass, i.e. when asymptotic stability in the design objectives listed under (i)-(iii) earlier in this section is replaced by the stronger requirement of stability along the pass.

Suppose that stability along the pass holds. Then as $k \rightarrow \infty$ we have for $0 \leq p \leq \alpha-1, x_{k+1}(p)=$ $x_{k}(p), y_{k+1}(p)=y_{r e f}(p)$ and hence

$$
\begin{aligned}
x_{\infty}(p+1) & =A x_{\infty}(p)+B u_{\infty}(p)+B_{0} y_{r e f}(p)+E w(p) \\
y_{r e f}(p) & =C x_{\infty}(p)+D u_{\infty}(p)+D_{0} y_{r e f}(p)+F w(p)
\end{aligned}
$$

Now introduce

$$
\begin{aligned}
& \hat{y}_{k}(p)=y_{k}(p)-y_{r e f}(p) \\
& \hat{x}_{k}(p)=x_{k}(p)-x_{\infty}(p) \\
& \hat{u}_{k}(p)=u_{k}(p)-u_{\infty}(p)
\end{aligned}
$$

Then using (31) and (1) we obtain

$$
\begin{array}{r}
\hat{x}_{k+1}(p+1)=A \hat{x}_{k+1}(p)+B \hat{u}_{k+1}(p)+B_{0} \hat{y}_{k}(p) \\
\hat{y}_{k+1}(p)=C \hat{x}_{k+1}(p)+D \hat{u}_{k+1}(p)+D_{0} \hat{y}_{k}(p)
\end{array}
$$

and in this so-called residual model the influence of the disturbance has been decoupled.

Suppose now that a stabilizing control law is required. Then this can be achieved by writing

$$
\hat{u}_{k+1}(p)=K_{1} \hat{x}_{k+1}(p)+K_{2} \hat{y}_{k}(p)
$$

or, equivalently,

$$
u_{k+1}(p)=u_{\infty}(p)+K_{1} x_{k+1}(p)-K_{1} x_{\infty}(p)+K_{2} y_{k}(p)-K_{2} y_{r e f}(p)
$$

and the task now is to construct $x_{\infty}(p)$ and $u_{\infty}(p)$ when $w(p)$ and $y_{r e f}(p)$ are known.

From the algebraic equation of $(31)$ for $y_{r e f}(p)$ we have that

$$
D u_{\infty}(p)=\left(I-D_{0}\right) y_{r e f}(p)-C x_{\infty}(p)-F w(p)
$$

Here we see again that not all $y_{r e f}(p)$ are available under this design. In particular, only those which ensure that

$$
\operatorname{rank}\left[D \mid\left(I-D_{0}\right) y_{\text {ref }}(p)-C x_{\infty}(p)-F w(p)\right]=\operatorname{rank}[D]
$$

are allowed. Suppose now that this condition holds (as is the case when $D$ is full row rank). Then we have that

$$
u_{\infty}(p)=D^{\sharp}\left(\left(I-D_{0}\right) y_{r e f}(p)-C x_{\infty}(p)-F w(p)\right)
$$

Also if $D$ is square and nonsingular, the pseudo inverse can be replaced by the matrix inverse and then

$$
u_{\infty}(p)=D^{-1}\left(\left(I-D_{0}\right) y_{r e f}(p)-C x_{\infty}(p)-F w(p)\right)
$$

Once $u_{\infty}(p)$ has been computed over $0 \leq p \leq \alpha-1$ then

$$
x_{\infty}(p+1)=\left(A-B D^{-1} C\right) x_{\infty}(p)+\left(B_{0}+B D^{-1}\left(I-D_{0}\right)\right) y_{r e f}(p)+\left(E-B D^{-1} F\right) w(p)
$$

(which is simply a 1D discrete linear systems state equation which can be computed given $d_{\infty}-$ the strong limit of the known pass state initial vector sequence) and the overall control law is of the form (32) where $K_{1}$ and $K_{2}$ are the control law matrices computed by the LMI method to give stability along the pass closed loop. 


\section{Conclusions}

This paper has developed substantial new results on the control of discrete linear repetitive processes. Here the focus is on control law design for stability and performance as opposed to the former alone in previous work. The design algorithms obtained have been illustrated on a model of physical process which has also been considered in the $2 \mathrm{D}$ systems literature. Work is currently in progress on both the in depth development/evaluation of these results and their extension. Output from this research will be reported in due course.

\section{References}

[1] Amann, N., Owens, D. H., and Rogers, E.: Predictive optimal iterative learning control, 1998, International Journal of Control, 69(2), pp. 203-226.

[2] Bachelier, O., and Bernussou, J., and de Oliveria, M. C., and Geromel, J. C.: Parameter dependent Lyapunov control design: numerical evaluation, 1999, Proceedings of 38th IEEE Conference on Decision and Control, pp. 293-297.

[3] Benton, S. E.: Analysis and Control of Linear Repetitive Processes, 2000, PhD Thesis, University of Southampton, UK.

[4] Boyd, S., Ghaoui, L.E., Feron, E. and Balakrishnan, V.: Linear Matrix Inequalities in Systems and Control Theory, 1994, SIAM Studies in Applied Mathematics. SIAM, Philadelphia.

[5] Edwards, J. B.: Stability problems in the control of multipass processes, 1974, Proceedings of The Institution of Electrical Engineers, 121(11), pp. 1425-1431.

[6] Fornasini, E., and Marchesini, G.: Doubly-indexed dynamical systems: state space models and structural properties, 1978, Mathematical Systems Theory, 12, pp. 59-72.

[7] Galkowski, K., and Rogers, E., and Xu, S.,. and Lam, J., and Owens, D.H.: LMIs - a Fundamental Tool in Analysis and Controller Design for Discrete Linear Repetitive Processes, 2002, IEEE Transactions on Circuits and Systems I: Fundamental Theory and Applications, 49(6), 768-778.

[8] Galkowski, K., and Rogers, E., and Wood, J., and Benton, S. E., and Owens, D. H.: 1D Equivalent Model and Related Approaches to the Analysis of Discrete Non-unit Memory Linear Repetitive Processes, 2002, Circuits, Systems, and Signal Processing, 21(6), pp. 525-534.

[9] Galkowski, K., and Lam, J., and Rogers, E., and Xu, S., and Sulikowski, B., and Paszke, W., and Owens, D. H.: LMI based stability analysis and robust controller design for discrete linear repetitive processes, 2003, International Journal of Robust and Nonlinear Control, 13(13), pp. $1195-1211$.

[10] Melkote, H., Cloke, B., and Agarwal, V.: Modeling and compensator design for self servo-writing in disk drives, 2003, Proceedings of American Control Conference, pp. 737-742.

[11] Roberts, P. D.: Two-dimensional analysis of an iterative nonlinear optimal control algorithm, 2002, IEEE Transactions on Circuits and Systems I: Fundamental Theory and Applications, 49(6) pp. 872-878.

[12] Roesser, R. P.: A discrete state space model for linear image processing, 1975, IEEE Transactions on Automatic Control, AC-20(1), pp. 1-10.

[13] Rogers, E. and Owens, D. H.: Stability Analysis for Linear Repetitive Processes, 1992, SpringerVerlag Lecture Notes in Control And Information Sciences Series, Vol 175, Berlin.

[14] Yamada, M., and Saito, O.: 2D model-following servo systems, 1990, Multidimensional Systems and Signal Processing, 10, pp. 71-91. 


\section{Summary of the changes made to MULT147-03}

The points raised by reviewer 1 have been addressed as follows.

1. A summary of the main steps, and reference to, the classical approach to stability analysis of these processes has been added - paragraph after (2) on page 2 .

2. The links between the stability theories for these processes and $2 \mathrm{D}$ linear systems has been clarified - first 3 paragraphs after Theorem 1.

3. The role of the $1 \mathrm{D}$ equivalent model has been clarified.

We also which to state that the example given disproves the idea proposed by this reviewer that $1 \mathrm{D}$ stability uniform with respect to $p$ is all that is required here. 